\title{
LINC81507 act as a competing endogenous RNA of miR-199b-5p to facilitate NSCLC proliferation and metastasis via regulating the CAV1/STAT3 pathway
}

Wei Peng ${ }^{1}$, Dan $\mathrm{He}^{2}$, Bin Shan ${ }^{3}$, Jun Wang ${ }^{1}$, Wenwen Shi', Wenyuan Zhao', Zhenzi Peng ${ }^{1}$, Qingxi Luo ${ }^{1}$, Minghao Duan ${ }^{1}$, Bin Li ${ }^{1,4}$, Yuanda Cheng ${ }^{5}$, Yeping Dong ${ }^{1}$, Faqing Tang ${ }^{2}$, Chunfang Zhang ${ }^{5}$ and Chaojun Duan ${ }^{1}$

\begin{abstract}
Lung cancer is the leading cause of cancer-related mortality worldwide. Recently, accumulating data indicate that long noncoding RNAs (LnCRNAs) function as novel crucial regulators of diverse biological processes, including proliferation and metastasis, in tumorigenesis. Lnc NONHSAT081507.1 (LINC81507) is associated with lung adenocarcinoma. However, its pathological role in non-small cell lung cancer (NSCLC) remains unknown. In our study we investigated the role of LINC81507 in NSCLC. The expression of LINC81507 was analyzed in 105 paired NSCLC tumor specimens and paired adjacent non-tumorous tissues from NSCLC patients by real-time quantitative PCR (RT-qPCR). Gain- and loss-offunction experiments were conducted to investigate the functions of LINC81507, miR-199b-5p and CAV1. Reduced expression of LINC81507 resulted in cell growth, proliferation, migration and epithelial-mesenchymal transition (EMT) in NSCLC cells, whereas ectopic overexpression of LINC81507 resulted in the opposite effects both in vitro and in vivo. Nuclear and Cytoplasmic fractionation assays showed LINC81507 mainly resided in cytoplasm. Bioinformatics analysis and dual-luciferase assays revealed that miR-199b-5p was a direct target of LINC81507 through binding Ago2. Mechanistic analysis demonstrated that miR-199b-5p specifically targeted the Caveolin1 (CAV1) gene, and LINC81507 inactivated the STAT3 pathway in a CAV1-dependent manner. Taken together, LINC81507 is decreased in NSCLC and functions as a sponge to miR-199b-5p to regulate CAV1/STAT3 pathway, which suggests that LINC81507 serve as a tumor suppressor and potential therapeutic target and biomarker for metastasis and prognosis in NSCLC.
\end{abstract}

\section{Introduction}

According to the Cancer Statistics released by American Cancer Society in 2018, lung cancer is not only the most frequently diagnosed cancer but also the leading cause of

\footnotetext{
Correspondence: Chaojun Duan (duancjxy@126.com)

'Department of Oncology, Xiangya Hospital, Central South University,

Changsha 410008, PR China

${ }^{2}$ Hunan Cancer Hospital, The Affiliated Tumor Hospital of Xiangya Medical

College, Central South University, Changsha 410008, PR China

Full list of author information is available at the end of the article.

Edited by E. Baehrecke
}

cancer death worldwide ${ }^{1}$. Non-small cell lung carcinoma (NSCLC) consist of almost $80 \%$ of lung cancer. NSCLC is the leading cause of cancer-related deaths in United States and in China ${ }^{1-3}$. Lack of obvious symptoms delays early diagnosis for lung cancer patients. Although the surgical comprehensive treatment has achieved great progress, 5year survival rate is still $<15 \%{ }^{4}$. Most NSCLC patients were diagnosed at an advanced stage. Accordingly, the molecular mechanisms' study of non-small cell lung cancer' proliferation and metastasis can provide guidance

\section{(c) The Author(s) 2019}

(c) (i) Open Access This article is licensed under a Creative Commons Attribution 4.0 International License, which permits use, sharing, adaptation, distribution and reproduction c. in any medium or format, as long as you give appropriate credit to the original author(s) and the source, provide a link to the Creative Commons license, and indicate if changes were made. The images or other third party material in this article are included in the article's Creative Commons license, unless indicated otherwise in a credit line to the material. If material is not included in the article's Creative Commons license and your intended use is not permitted by statutory regulation or exceeds the permitted use, you will need to obtain permission directly from the copyright holder. To view a copy of this license, visit http://creativecommons.org/licenses/by/4.0/. 
for development of diagnosis, prognostic and therapeutic strategies. Emerging evidence shows that although long noncoding RNAs (LncRNAs) lack the protein coding potential, they can control transcription, translation and protein function through various mechanisms ${ }^{5}$. More and more attentions had been focused on LncRNAs, especially in cancer.

LncRNAs are a diverse class of RNAs whose length is longer than 200 nucleotides ${ }^{6}$. Recently, Iyer and his colleges showed that there are about 60,000 noncoding RNAs in the human genome while almost 42,000 are IncRNAs ${ }^{7}$. However, the total number is still climbing. About $80 \%$ of $\operatorname{lncRNAs}$ are not fully elaborated. LncRNAs could engage in various biological processes fuse in every branch of life. LncRNAs can act as scaffolds, decoys and signal transducers via cis and trans manners ${ }^{8-10}$. LncRNAs have the potential in NSCLC diagnosis and prognosis $^{11}$. Moreover, lncRNA-P53RRA can promote ferroptosis and apoptosis in cancer via sequestration of $\mathrm{p} 53^{12}$. Several lines of evidence emphasizes that lncRNAs function as competitive endogenous RNA (ceRNA) for miRNAs in NSCLC ${ }^{13,14}$. OIP5-AS1 can promote lung cancer cells' proliferation by targeting miR-378a-3p ${ }^{15}$. LINC00339 sponges miR-145 and interferes FOXM1 to facilitate NSCLC tumorigenesis ${ }^{16}$. MALAT1 activates Akt/mTOR signaling via targeting miR-206 to accelerate NSCLC cells migration and invasion ${ }^{17}$.

Lnc NONHSAT081507.1 (LINC81507) was first identified by our team using Agilent Human LncRNA Array ${ }^{18}$. We found that LINC81507 expression was lower in NSCLC including adenocarcinoma and squamous cell carcinoma when compared with adjacent normal tissues, especially in the tumors with lymph node metastasis. We also found that LINC81507 acts as a ceRNA for miR-199b-5p through direct binding and interferes miR-199b-5p-mediated regulation of CAV1, reduces migration and invasion. In addition, the expression of LINC81507 were positively correlated with 3-year survival. Thus, LINC81507 is an independent predictor for the overall survival in NSCLC. In summary, our findings demonstrate tumor suppressive functions of LINC81507 in NSCLC progression and LINC81507 can serve as a therapeutic target and potential biomarker for diagnosis and prognosis NSCLC.

\section{Methods}

\section{Patient and specimens}

The total 105 cancer tissues and paired adjacent nontumor tissues (ANLTs, which obtained at least $5 \mathrm{~cm}$ away from the tumor edge) were obtained from patients with NSCLC during operation at the Department of Thoracic Surgery, Xiangya Hospital of Central South University (Changsha, China) from January 2012 to July 2016. Clinicopathological TNM staging was judged according to the 8th lung cancer TNM classification criteria. The clinicopathological characteristics of 105 patients were summarized in Table 1 . All the fresh specimens were rapidly frozen in liquid nitrogen, and then stored in $-80^{\circ} \mathrm{C}$ refrigerator.

\section{Cell line}

Five human lung cancer cell lines (PC-9, H1299, A549, H1975 and Calu-1) were purchased from Chinese Academy of Sciences Cell Bank (Shanghai, China). SK-MES-1 were purchased from Shanghai Institutes for Biological Sciences, CAS. PC-9, H1299, A549, H1975, Calu-1 and human bronchial epithelium cells (HBE) were cultured in RPMI 1640 medium containing $10 \%$ fetal bovine serum (FBS) and $1 \%$ penicillin-streptomycin $(\mathrm{P} / \mathrm{S})$ at $37^{\circ} \mathrm{C}$ and $5 \% \mathrm{CO}_{2}$, SK-

Table 1 Correlation analysis of clinicopathological features between LINC 81507 and NSCLC patients

\begin{tabular}{|c|c|c|c|c|}
\hline \multirow[t]{2}{*}{ Characteristics } & \multirow[t]{2}{*}{ Number } & \multicolumn{2}{|c|}{$\begin{array}{l}\text { LINC81507 } \\
\text { expression }\end{array}$} & \multirow[t]{2}{*}{$P$ value } \\
\hline & & Low $(n)$ & High $(n)$ & \\
\hline \multicolumn{5}{|l|}{ Age (years) } \\
\hline$\leq 60$ & 40 & 30 & 10 & 0.965 \\
\hline$>60$ & 65 & 49 & 16 & \\
\hline \multicolumn{5}{|l|}{ Pathological type } \\
\hline Adenocarcinoma & 83 & 60 & 23 & 0.174 \\
\hline Squamous cell carcinoma & 22 & 19 & 3 & \\
\hline \multicolumn{5}{|l|}{ Gender } \\
\hline Male & 64 & 45 & 19 & 0.144 \\
\hline Female & 41 & 34 & 7 & \\
\hline \multicolumn{5}{|l|}{ Tumor invasion } \\
\hline T1 & 12 & 11 & 1 & 0.003 \\
\hline $\mathrm{T} 2$ & 70 & 56 & 14 & \\
\hline T3 & 13 & 9 & 4 & \\
\hline $\mathrm{T} 4$ & 10 & 3 & 7 & \\
\hline \multicolumn{5}{|l|}{ Lymph node metastasis } \\
\hline NO & 69 & 60 & 9 & $<0.01$ \\
\hline N1 & 22 & 13 & 9 & \\
\hline $\mathrm{N} 2+\mathrm{N} 3$ & 14 & 6 & 8 & \\
\hline \multicolumn{5}{|l|}{ Distant metastasis } \\
\hline MO & 90 & 72 & 18 & 0.006 \\
\hline M1 & 15 & 7 & 8 & \\
\hline \multicolumn{5}{|l|}{ TNM stage } \\
\hline । & 47 & 34 & 13 & $<0.05$ \\
\hline$\|$ & 40 & 36 & 4 & \\
\hline$I I I+I V$ & 18 & 9 & 9 & \\
\hline
\end{tabular}


Table 2 Primer sequence

\begin{tabular}{|c|c|}
\hline \multicolumn{2}{|l|}{ Lnc NONHSAT081507.1 } \\
\hline $\mathrm{F}$ & TACTGTTTCCAAACTGGACACTGGAGAATATTCC \\
\hline R & TITTTAAGTTAGTTTATACAAATGCCTATGAAATCAAATTAGTGACCA \\
\hline miRNA 199b-5p & CGCGCGACCCAGTGTTTAGACTATCTGTTC \\
\hline miRNA 30e-5p & GCCGCGTGTAAACATCCTTGACTGGAAG \\
\hline miRNA 106a & GCGAAAAGTGCTTACAGTGCAGGTAG \\
\hline \multicolumn{2}{|l|}{ CAV1 } \\
\hline $\mathrm{F}$ & CATCCCGATGGCACTCATCTG \\
\hline $\mathrm{R}$ & TGCACTGAATCTCAATCAATCAGGAAG \\
\hline \multicolumn{2}{|l|}{ CAV1-shRNA } \\
\hline 1 & GACGTGGTCAAGATTGACTTTTCAAGAGAAAGTCAATCTTGACCACGTCTITTIT \\
\hline 2 & GCCACCTTCACTGTGACGAAATTTCAAGAGAATTTCGTCACAGTGAAGGTGGTTTIT \\
\hline 3 & GACCCACTCTTTGAAGCTGTTTTCAAGAGAAACAGCTTCAAAGAGTGGGTCTTTTIT \\
\hline \multicolumn{2}{|l|}{ 36B4 } \\
\hline $\mathrm{F}$ & CAACCCAGCTCTGGAGAAAC \\
\hline $\mathrm{R}$ & GTGAGGTCCTCCTTGGTGAA \\
\hline \multicolumn{2}{|l|}{ U6 } \\
\hline $\mathrm{F}$ & CGAACGATACAGAGAAGATTAGC \\
\hline $\mathrm{R}$ & TGGAACGCTTCACGAATTTGCG \\
\hline \multicolumn{2}{|l|}{ Actin } \\
\hline $\mathrm{F}$ & CCTGTACGCCAACACAGTGC \\
\hline $\mathrm{R}$ & ATACTCCTGCTTGCTGATCC \\
\hline \multicolumn{2}{|l|}{ GAPDH (nuclear) } \\
\hline $\mathrm{F}$ & GGGAGCCAAAAGGGTCAT \\
\hline R & GAGTCCTTCCACGATACCAA \\
\hline \multicolumn{2}{|l|}{ U6 (cytoplamic) } \\
\hline $\mathrm{F}$ & CTCGCTTCGGCAGCACA \\
\hline $\mathrm{R}$ & AACGCTTCACGAATTTGCGT \\
\hline \multicolumn{2}{|l|}{ Probe of FISH } \\
\hline \multicolumn{2}{|l|}{1 TTCCA GTAAG TATAC TTTCC TACAA TGCAT GTCTT TCTCC } \\
\hline \multicolumn{2}{|l|}{2 AGGTT TAATT GGCTC ACGGT TCTGC AAGCT GTACA ATCCT } \\
\hline 3 ACTCT ATCAA CATCC CACTT CACAC TTCAT ACCTA CTTGT & \\
\hline
\end{tabular}

MES-1 was maintained in MEM medium containing $10 \%$ FBS and P/S. All cell lines were passaged $<10$ times after the initial revival from frozen stocks. All cell lines were authenticated prior to use by short tandem repeat profiling.

\section{RNA extraction and real-time quantitative polymerase} chain reaction

Total RNA was isolated from cells and frozen tissue specimens using TRIzol reagent (Invitrogen, Carlsbad, CA). cDNA was generated using GoScript Reverse
Transcription System (Promega, USA). The RNA levels were quantified using SYBR Green qPCR Mix (GeneCopoeia, USA). 36B4, U6 and Actin were used to normalize LncRNAs, miRNAs and mRNAs. The primers were listed in Table 2.

\section{Fluorescent in situ hybridization (FISH) assays}

In situ detection of IncRNA was performed in lung cancer tissues using BOSTER kit. Fluorescence-labeled probes for LINC81507 were presented in Table 2. 


\section{Nuclear and cytoplasmic fractionation}

Nuclear and cytoplasmic fractionation using PARIS ${ }^{\text {mix }}$ Kit (Invitrogen $^{\mathrm{nt}}$, AM1921) were performed in A549 and Calu-1 cells. The RNA expression was measured using RT-qPCR.

\section{Cell transfection, lentivirus production, and transduction}

The SMARTer ${ }^{\text {Ta }}$ RACE cDNA kit (Takara) was used to obtain the full sequence of LINC81507. The pcDNA3.1 vector for LINC81507 overexpression and negative control were constructed by Shanghai Genechem. Cells were harvested for analysis at $48 \mathrm{~h}$ after transfection. After lentivirus transduction, PC-9, A549, and Calu-1 cells were treated with $1 \mathrm{mg} / \mathrm{ml}$ puromycin to establish stable cell lines. The hsa-miR-199b-5p mimic, hsa-miR-199b-5p inhibitor, and negative control (NC) oligonucleotides were purchased from Ruibio (Guangzhou, China). The pcDNA3.1-CAV1, pcDNA3.1 vectors, pLent-CAV1shRNA, and pLent vectors were purchased from Vigene Biosciencces (USA). The cell transfections were performed using lipofectamine 3000 (Invitrogen).

\section{Cell counting kit-8 assays}

Cell proliferation was monitored using cell counting kit8 (CCK8) (Beyotime Biotechnology). A549, PC-9, and Calu- 1 cells $\left(3 \times 10^{3} /\right.$ well $)$ were seeded in 96 -well plates. Cell proliferation was documented on 24, 48, 72, and $96 \mathrm{~h}$. The experiments were repeated three times.

\section{Colony formation assays}

After transfection, A549, PC-9 $\left(2 \times 10^{2} /\right.$ well $)$ and Calu-1 cells $\left(7 \times 10^{2} /\right.$ well $)$ were cultured in 6 -well plates for 14 days. Paraformaldehyde fix solution (4\% PFS) and $0.1 \%$ crystal violet (Beyotime Biotechnology) were added into 6-well plates. Visible cell colonies were then counted.

\section{5-ethylnyl-2'-deoxyuridine (EdU) incorporation assay}

EdU assay was performed in Cell-Light ${ }^{\mathrm{Tm}}$ EdU Apollo 488 In Vitro Kit (Ruibio, China). A549, PC9, and Calu-1 cells $\left(1 \times 10^{4}\right)$ were incubated in 96-well plates after plasmid transfection. Hoechst 33,342 was used to identify nucleic acid in these three cell lines under a fluorescence microscope.

\section{Migration and invasion assays}

The cells $\left(4 \times 10^{4} / 100 \mu \mathrm{l}\right)$ in serum-free media were seeded into the upper chambers that were covered with (invasion) or without (migration) Matrigel (BD, USA). The lower chambers contained $600 \mu \mathrm{l} 1640$ medium containing antibiotics and 10\% FBS. After $36 \mathrm{~h}$, the cells that had migrated or invaded through the upper chamber were dyed with $4 \%$ PFS and $0.1 \%$ crystal violet. Then, an inverted microscope (Canon, Japan) was used to count and capture these images.

\section{Gelatin zymography}

Gelatin zymography was a classical method to detect gelatinases in the supernatants or cell lysates ${ }^{19}$. The supernatants and cell lysates of A549 and Calu-1 cells (which contained $10 \mu \mathrm{l}$ supernatants/proteins of cell lysate and $10 \mu \mathrm{l}$ native gel loading buffer) were loaded on a gelatin (G-8061, Solarbio) containing 10\% SDS polyacrylamide gels followed by electrophoresis for $140 \mathrm{~V}-100 \mathrm{~min}$ at $4^{\circ} \mathrm{C}$. Triton X-100 was used to wash the gels to renature MMP proteins, followed by incubation for $36 \mathrm{~h}$ at $37^{\circ} \mathrm{C}$. Gels were stained with Coomassie blue staining for $90 \mathrm{~min}$ and then tarnished for $60 \mathrm{~min}$ until bands visible.

\section{Bioinformatics methods}

Starbase, NONCODE 2.0, TANRIC and LNCipedia were used to predict LINC81507 potential target microRNA. Targetscan, miRanda and mirBase were used to predict potential target gene, combined with the literature and through the test screening, miR-199b-5p and CAV1 were selected for further studies.

\section{Luciferase reporter assay}

293T cells were seeded in a 24-well plates. Twenty-four hours after co-transfected with miR-199b-5p mimics/ inhibitors and the corresponding luciferase reporter vectors (LINC81507 and CAV1 as indicated), dual luciferase reporter assay system (Promega, Madison, WI, USA) were applied.

\section{RNA-binding protein immunoprecipitation assay}

Magna RNA immunoprecipitation (RIP) kit (Millipore, USA) was conducted to finish RIP assay. A549 cell lysate was incubated in RIP buffer that contained magnetic beads and was conjugated with human anti-Ago2 antibody, input or normal rabbit IgG were included as negative control. Proteinase $\mathrm{K}$ was used in purifying the immunoprecipitated RNAs. RT-qPCR were performed to detect the binding of target LINC81507 and miR-199b-5p.

\section{Xenografted tumor model}

BALB/c-nu nude male mices (age of 3-4 weeks, 16-18 g) were obtained from the Hunan Slack King of Laboratory Animal Co., Ltd (Changsha, China). For the in vivo tumor proliferation assay, $1 \times 10^{7}$ A549 cells transfected with LINC81507-overexpression (LINC81507-OE) or LINC81507-control (LINC81507ctrl) were injected subcutaneously into nude mice (6 per group). The tumor volume was monitored and recorded once a week. Tumor volume was calculated as follows: volume $=$ length $\times$ width $^{2} / 2.25$ days later, the mices were sacrificed. For in vivo metastasis assays, $1 \times 10^{6}$ A549 ${ }^{\text {LINC81507-OE }}$ cells and A549 ${ }^{\text {LINC81507-ctrl }}$ cells were injected intravenously into the tail vein of mice. Each 
animal was injected with 2 nmol MMP Sense 750 FAST (PerkinElmer, USA) 60 days later. At $12 \mathrm{~h}$ after injection, mices were imaged by Fluorescence Molecular Tomography system (PerkinElmer, USA) and analyzed using corresponding software (True Quant, PerkinElmer, USA). All mices were sacrificed, and metastatic nodules were calculated. Then, lung tissues were subjected to immunohistochemical analysis.

\section{Western blot assay}

The total proteins were obtained from cells and then quantified using BCA method. These protein samples were separated through sodium dodecyl sulfatepolyacrylamide gel electrophoresis (SDS-PAGE), and then transferred to a methanol activated PVDF membrane. After blocking with skim milk at the room temperature for $1 \mathrm{~h}$, the membrane was incubated in primary antibodies in a proper dilution overnight at $4{ }^{\circ} \mathrm{C}$. After that, the membranes were incubated with rabbit or mouse secondary antibodies for $1 \mathrm{~h}$ at the room temperature and then visualized using chemiluminescence reagents.

\section{Immunohistochemistry staining (IHC)}

IHC analysis was performed on a 60 paraffin-embedded NSCLC tissue microarrays, using anti-CAV1 (1:500, ab32577, Abcam). Then, we performed HE and IHC in the lungs, using the following primary antibodies, anti-Ecadherin (1:500, ab76055, Abcam), anti-N-cadherin (1:200, ab18203, Abcam), anti- $\beta$-catenin (1:200, ab32572, Abcam), anti-Vimentin (1:200, ab92547, Abcam).

\section{Statistical analysis}

All statistical analyses were performed using SPSS 17.0 software and Graph Pad Prism. These experiments were performed independently at least three times. Student's $t$-test or one-way ANOVA was used to analyze the mean between different groups. LINC81507 expression and clinicopathological variables was calculated by the $X$ test or Fisher's exact test. Survival curves were established using the Kaplan-Meier method and compared by the log-rank test. $P<0.05$ was considered statistically significant.

\section{Results}

Lower expression of LINC81507 was associated with advanced TNM staging and metastasis of NSCLC

We previously reported that LINC81507 was differentially expressed in $\mathrm{ADC}^{18}$. According to NONCODE 2.0 and LNCipedia database annotation, LINC81507 is located on chr21: 27074489-27082491 and transcribed into a $946 \mathrm{nt}$ long lncRNA ${ }^{18}$ (additional file. 2a). In this study, we finished the RT-qPCR in 105 NSCLC tumor tissues and paired adjacent normal lung tissues to measure the expression of LINC81507. As shown in Fig. 1a, we compared LINC81507 expression in NSCLC tissues with ANLTs. We found that the expression of LINC81507 in tumor tissues was markedly decreased in NSCLC patients, especially in tumor tissues with lymph node metastasis $(P$ $<0.01$ ) (Fig. 1b). The same trend was observed in TNM stage $(P<0.05)$ (Fig. 1c). Table 1 showed TNM stage of all tumors. The correlated clinicopathological analysis revealed that reduced expression of LINC81507 was associated with lymph node metastasis $(P<0.01)$, distant metastasis $(P<0.01)$, and tumor invasion $(P<0.01)$. Collectively, these clinical datas suggest that the reduced expression of LINC81507 in NSCLC may be involved in NSCLC progression and metastasis. Kaplan-Meier analysis and Log-rank test revealed the correlation between LINC81507 expression and 3-year overall survival. The median survival time for the low LINC81507 expression group was shorter than the high expression group. As shown in Fig. 1d, the high expression of LINC81507 was linked to a better 3-year OS in patients with NSCLC than the low expression group $(P<0.05)$.

\section{LINC81507 inhibits proliferation, EMT, and metastasis in vivo}

Because of reduced expression of LINC81507 in lung cancer, we further explored the role of LINC81507 in proliferative and metastatic potential in mice. First, we performed the RT-qPCR in lung cancer cell lines and normal human lung cell line HBE (Fig. 1e). A549, PC-9, and Calu-1 exhibited low expression of LINC81507 and were selected for further studies. Then, we construct the LINC81507 overexpression (LINC81507-OE) and the matching LINC81507 control (LINC81507-ctrl) cell lines for further research (additional file. 1a). Among these cell lines, the A549 variants were chosen for mouse model.

As we expected, in mouse model, bioluminescence imaging showed that animals of the A549 ${ }^{\text {LINC81507-ctrl }}$ group exhibited more lung metastatic tumors. Increased metastasis to the lung was confirmed by histological analysis (Fig. 2e). In contrast to the control group, A549 $9^{\text {LINC81507-OE }}$ group showed less and smaller lung metastatic nodules (Fig. 2d). These results indicate that LINC81507 inhibited metastasis in vivo. In mouse subcutaneously tumorigenicity model, the average tumor volume of A549 $9^{\mathrm{LINC}} 1507-\mathrm{OE}$ group was obviously smaller than the tumors volume in the A549 $9^{\text {LINC81507-ctrl }}$ group ( $P$ $<0.01$ ) (Fig. 2a). The growth-curve of subcutaneous tumor volume was evaluated and a distinct difference was observed (Fig. 2b). The percentage of Ki-67 positive cells was decreased in A549 ${ }^{\mathrm{LINC} 81507-\mathrm{OE}}$ group compared with A549 ${ }^{\text {LINC81507-ctrl }}$ group (Fig. 2c). These results provided 

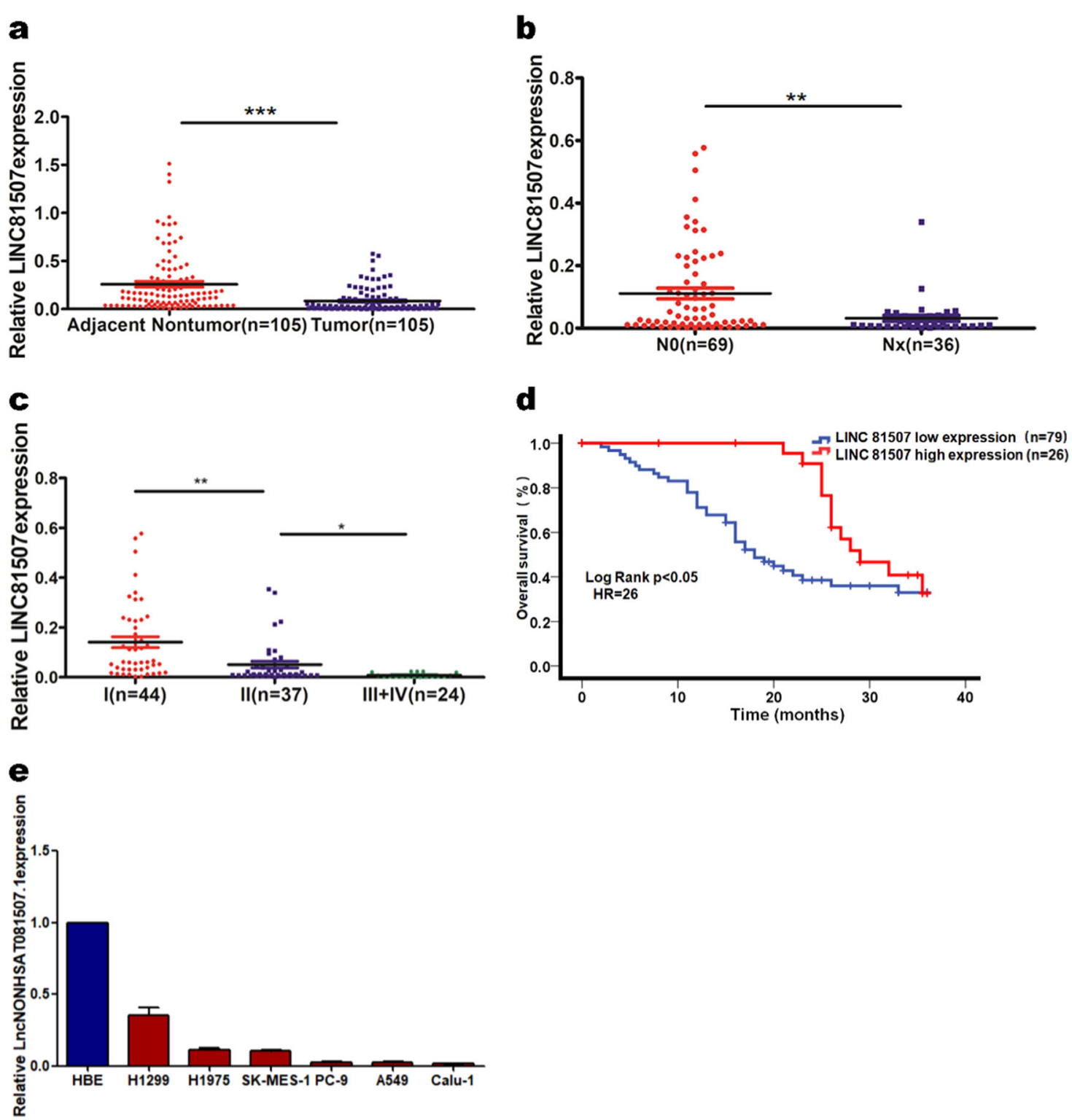

Fig. 1 LncRNA-LINC81507 expression profile and survival curve in NSCLC. a LINC81507 expression were evaluated using RT-qPCR in 105 pairs of NSCLC tissues and the matched adjacent normal tissues. b LINC81507 expression in NSCLC patients with/without node metastasis. 1+2+3 Nx means the patients who have node metastasis. c LINC81507 expression in NSCLC patients whose stages were I/II/III+IV. d Kaplan-Meier survival curve indicated the high LINC81507 expression is correlated with well survival rates. e LINC81507 expression in NSCLC cell lines and normal human bronchial epithelial cell line. Data were represented as the mean \pm SEM of three independent experiments. ${ }^{*} P<0.05,{ }^{* *} P<0.01,{ }^{* * *} P<0.001$

further evidence that LINC81507 inhibits proliferation in NSCLC.

Recent evidence showed that EMT is an important process, especially in tumor metastasis ${ }^{20-22}$. Thus, we also examined the expression of the related EMT marker proteins in lungs by IHC assay. The expression of Ecadherin, an epithelial marker, was increased in the A549 ${ }^{\mathrm{LINC} 81507-\mathrm{OE}}$ tumors, while the mesenchymal marker $\mathrm{N}$-cadherin, vimentin, and $\beta$-catenin were significantly decreased (Fig. 2e). These results indicate that LINC81507 inhibits proliferation and EMT in vivo.

\section{LINC81507 overexpression inhibits lung cancer cells' proliferation, migration, and invasion in vitro}

Since the expression of LINC81507 is downregulated and correlates with NSCLC progression and metastasis, gain-of-function experiments were used to determine the role of LINC81507 in NSCLC cells' proliferation and 
$\mathbf{a}$
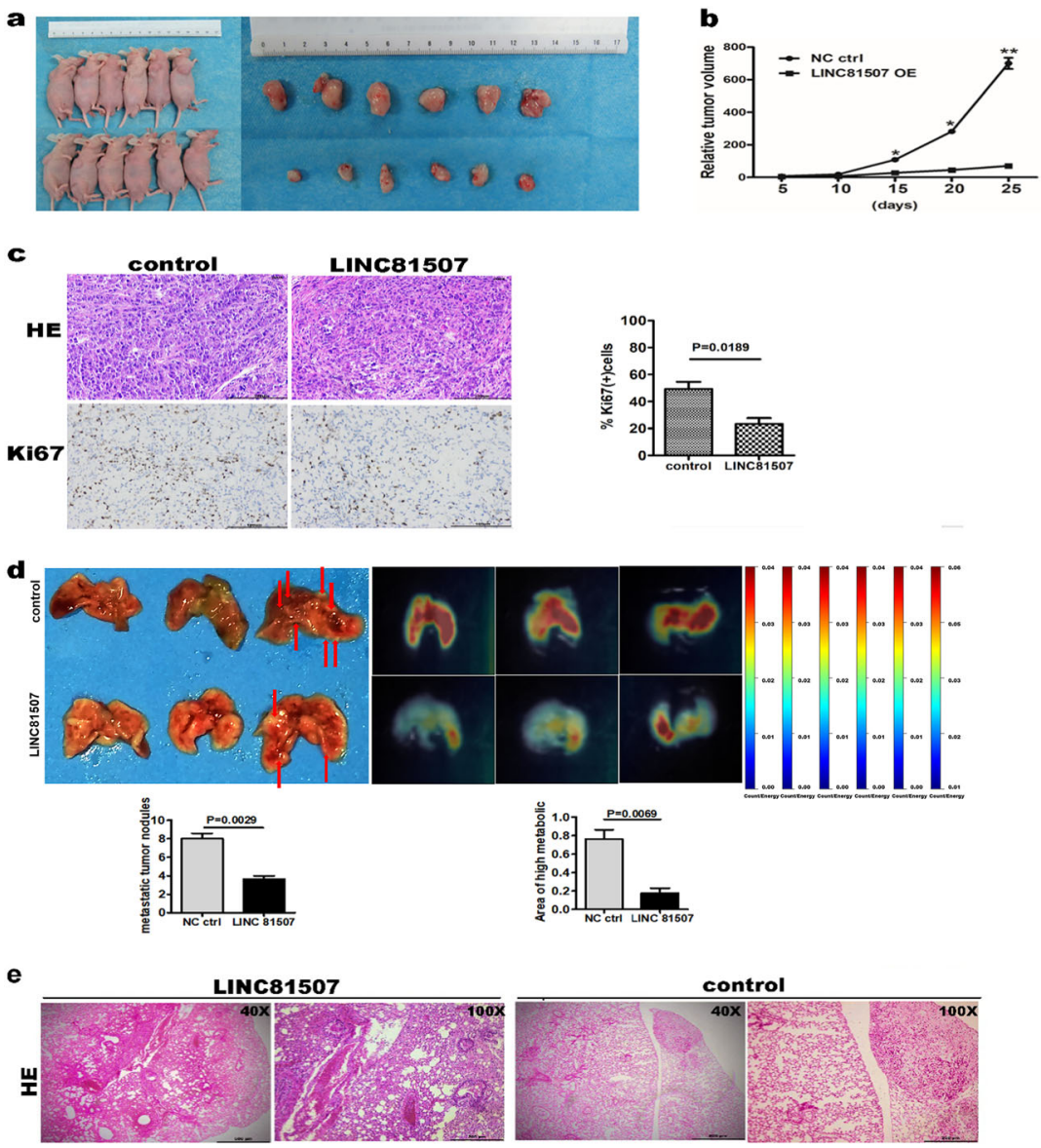

e
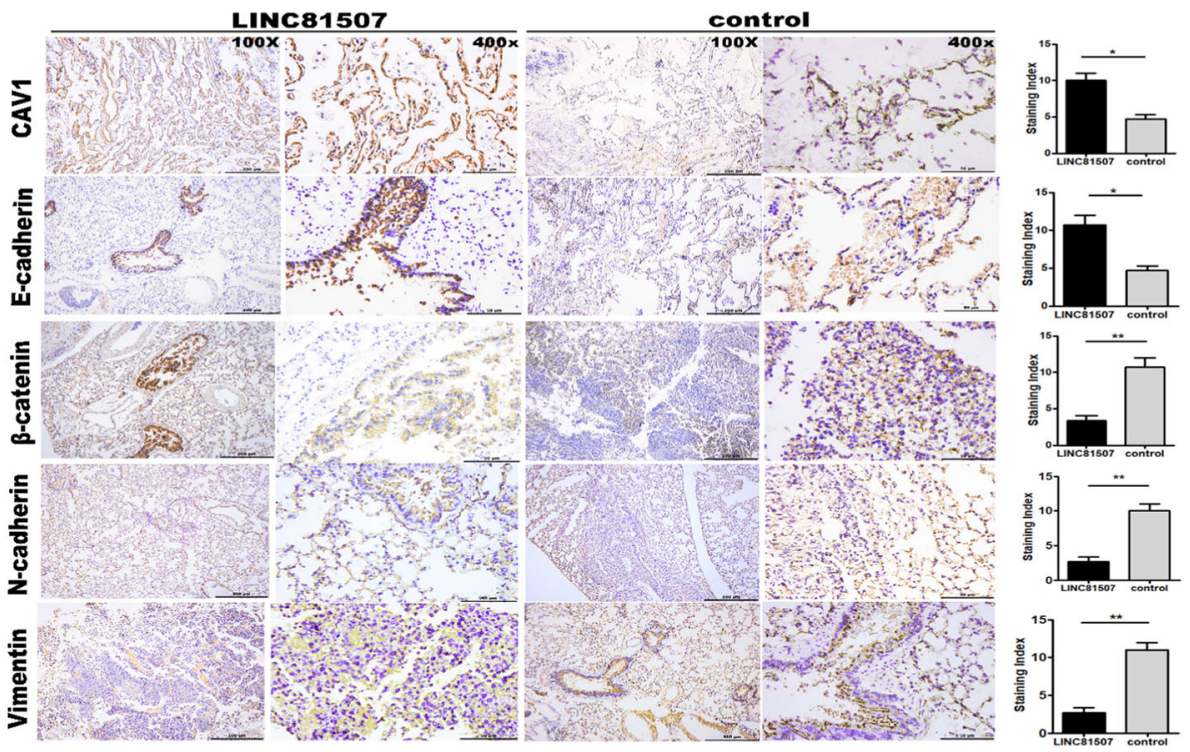

Fig. 2 LINC81507-overexpression inhibits proliferation and metastasis in vivo. a Representative images of the xenograft tumors. $\mathbf{b}$ The relative volumes of tumors were analyzed. c Representative images of HE and immunohistochemical staining for Ki67 expression in tumor tissues, $\times 20$ are shown. d LINC81507-overexpression inhibits A549 cells metastasis in vivo $(n=3)$. Representative photographs of gross lungs (left), with arrows pointing to lung surface tumor nodules, and the bioluminescent change in A549 ${ }^{\text {LINC81507-OE }}$ group was significantly decreased compared with the vector control (right), the relative tumor nodules and high metabolic area were showed. The quantitation of lung metastasis was assessed by bioluminescence measurements. e H\&E-stained section of lung, with original magnification: $\times 4, \times 10$ are shown. Micrograph indicates the magnified morphology of tumor tissues. Representative images of immunohistochemical staining for CAV1, E-cadherin, N-cadherin, $\beta$-catenin, and Vimentin expression in mouse metastasis lung tissues compared with normal mouse lung tissues (in $\times 10$ and $\times 40$ ). Data were represented as the mean \pm SEM of three independent experiments. ${ }^{*} P<0.05,{ }^{* *} P<0.01,{ }^{* * *} P<0.001$ 


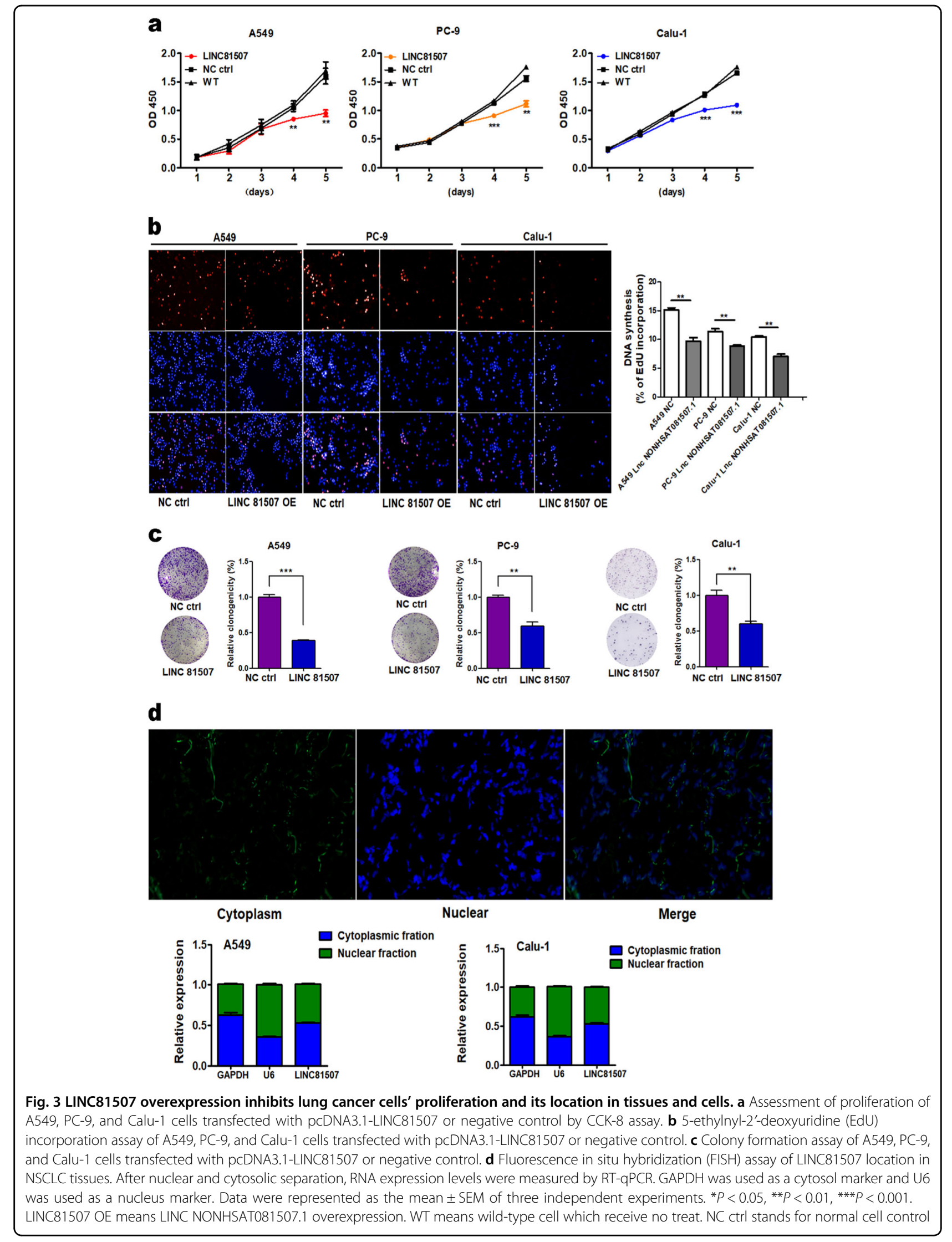




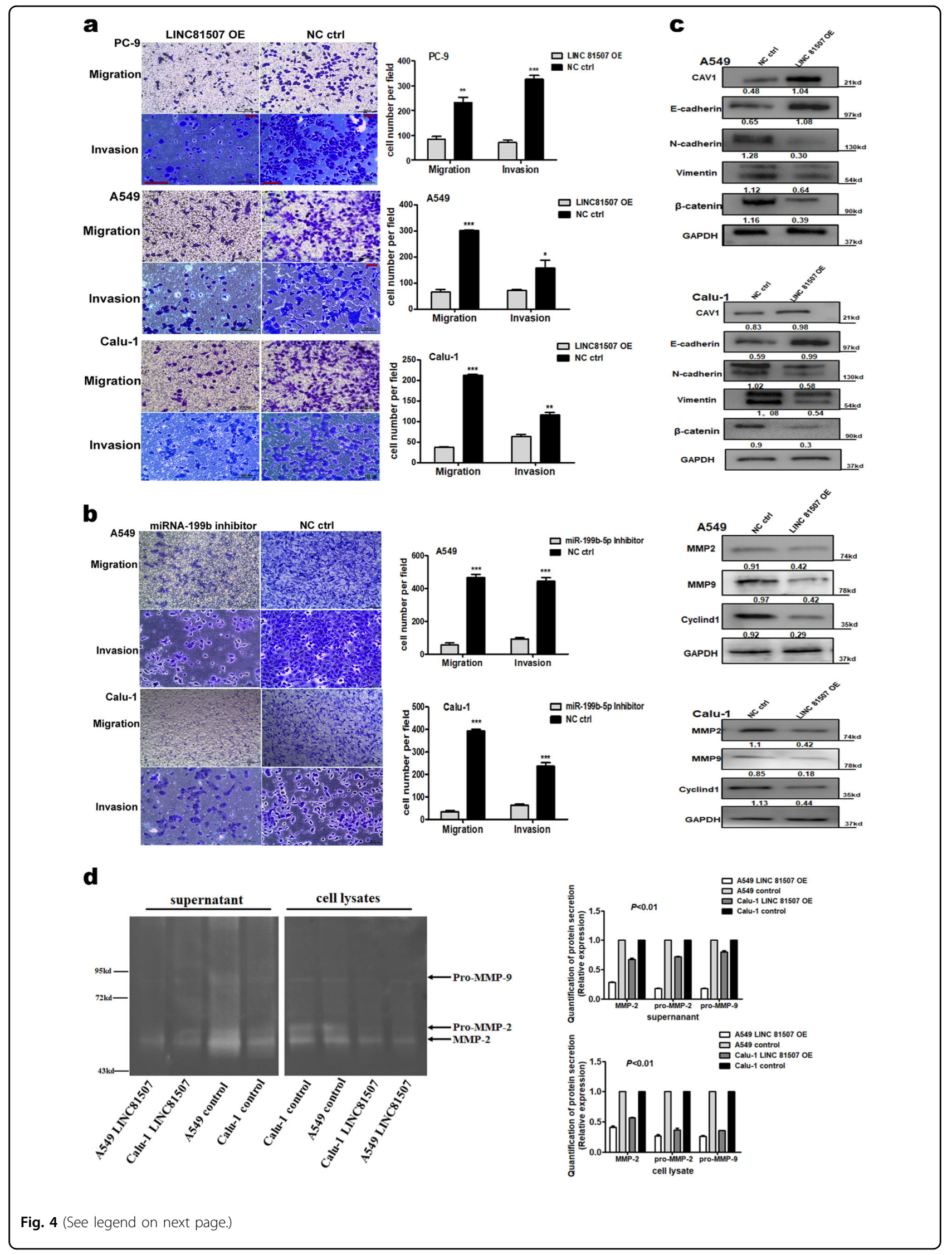


(see figure on previous page)

Fig. 4 Ectopic expression of LINC81507 inhibits lung cancer cells' migration and invasion. a Migration and invasion assay of A549, PC-9, and Calu-1 cells transfected with pcDNA3.1-LINC81507 or negative control. b Migration and invasion assay of A549 and Calu-1 cells transfected with miRNA-199b inhibitor or negative control. c In A549 and Calu-1 cells with LINC81507 overexpression, the protein levels of CAV1 and E-cadherin were significantly increased compared with the control, while N-cadherin, Vimentin, and B-catenin significantly decreased. Related expression of MMP2, MMP9, and Cyclin D1 protein were increased in LINC81507-overexpression group by western blot analysis. d Representative illustration of a zymography gel for detection of latent and active gelatinase levels ((pro-)MMP-2 and -9). The analysis was performed in culture supernatants and cell lysates of A549 and Calu-1 cells in serum-free medium for $24 \mathrm{~h}$. Samples were loaded on an electrophoresis gel containing gelatin. The detected bands of MMP-2/pro-MMP-2 and the bands showed decreasing expression with LINC81507 overexpression. The same trend in Pro-MMP-9, while MMP-9 was not detectable by gelatin zymography. Data were represented as the mean \pm SEM of three independent experiments. ${ }^{*} P<0.05$, ${ }^{* *} P<$ $0.01,{ }^{* * *} P<0.001$. miRNA-199b short for miRNA-199b-5p

migration. To elucidate the relationship between LINC81507 and proliferation or metastasis of NSCLC, we performed CCK8 assay (Fig. 3a), colony formation (Fig. 3c), EdU (Fig. 3b), migration, and invasion (Fig. 4a) assays in A549, PC-9, and Calu-1 with LINC81507-OE or control. These findings indicated that overexpression of LINC81507 inhibited lung cancer cell proliferation, migration and invasion. We examined protein levels of Ecadherin, $\mathrm{N}$-cadherin, vimentin, and $\beta$-catenin using western blot. Their alteration was consistent with the findings from the in vivo experiments (Fig. 4c). In addition, MMP-2, MMP-9, and Cyclin D1 that act as key players in tumor cell dissemination ${ }^{23}$ and proliferation ${ }^{24}$ in cancer were decreased in LINC81507-OE group (Fig. 4c). Moreover, overexpression of LINC81507 decreased gelatinases as shown by gelatin zymography (Fig. 4d). The lower levels of latent MMP-2 (pro-MMP-2) and latent MMP-9 (pro-MMP-9) were observed in LINC81507-OE group in both supernatants and cell lysates. Overall, these findings indicate that LINC81507 inhibited lung cancer cell's proliferation, migration and invasion, and EMT.

\section{LINC81507 functions as a miR-199b-5p sponge in lung cancer cells}

Using RACE (additional file 2a), we obtained full sequence of LINC81507. Furthermore, we performed FISH assay in tissues and cytoplasmic/nuclear fractionations and showed that LINC81507 was mainly located in cytoplasm (Fig. 3d). Lots of research showed LncRNAs could function as ceRNAs to regulate target gene expression by binding to miRNAs ${ }^{13}$. Using bioinformatics database miRbase and TANRIC ${ }^{25}$ that was based on correlation coefficient, we identified top three negatively correlated miRNAs (miR-199b-5p, miR-30e and miR106a) (additional file 6). Among these miRNAs, we further verified that miR-199b-5p expression was significantly increased in NSCLC tissues and NSCLC cells (Fig. 5a, b). We noticed an inverse correlation between miR-199b-5p and LINC81507 in transfected A549, PC-9 and Calu-1 cells (Fig. 5c). Luciferase reporter assay was used to examine the effects of LINC81507 on miR-199b-5p- regulated gene expression. The binding sites of miR-199b5p on LINC81507 are indicated in Fig. 5h. The miR-199b$5 p$ predicted binding site of LINC81507 (LINC81507WT) and a mutated miR-199b-5p binding site of LINC81507 (LINC81507-MUT) were cloned into a reporter plasmid. Then, we carried out dual-luciferase assays to determine whether LINC81507 altered the reporter controlled by miR-199b-5p. Co-transfection of miR-199b-5p mimic and LINC81507-WT decreased the luciferase activity, whereas co-transfection of LINC81507WT and miR-ctrl and co-transfection of miR-199b-5p mimic and LINC81507-MUT did not change the luciferase activity (Fig. 5h). Taken together, our data suggested a reciprocal interaction between LINC81507 and miR-199b-5p. RIP assay showed that LINC81507 and miR-199b-5p were co-immunoprecipitated through binding to Ago2 simultaneously, but not the IgG (Fig. 5i). Consistently, in migration assays, miR-199b-5p exhibited pro-migratory activity (Fig. 4b). Moreover, inhibition of migration by LINC 81507 was reversed by co-transfection of miR-199b-5p mimic (Fig. 5f). Taken together, these findings suggest that LINC81507 function as a miR-199b5 p sponge in lung cancer cells.

\section{LINC81507 suppresses NSCLC migration and metastasis by interfering miR-199b-5p/CAV1 axis}

MicroRNAs exert their diverse biological functions mainly by degrading mRNA or suppressing mRNA translation $^{26}$. To identify the signaling pathways that are involved in LINC81507/miR-199b-5p regulation of NSCLC proliferation and metastasis, integrated bioinformatical analysis (miRbase, Targetscan and TANRIC) were performed by our team. We identified four potential miR199b-5p target mRNAs, Caveolin1(CAV1), ITGA3, GRP48, and HIF1. We performed RT-qPCR to examine these four mRNAs expression in NSCLC tissues. Among these mRNAs, we further verified that CAV1 expression was significantly decreased in NSCLC tissues (Fig. 5e). These results suggest miR-199b-5p has the potential to bind and reduce the expression of CAV1. Thus, we performed luciferase reporter assay (Fig. 5h) and rescue 


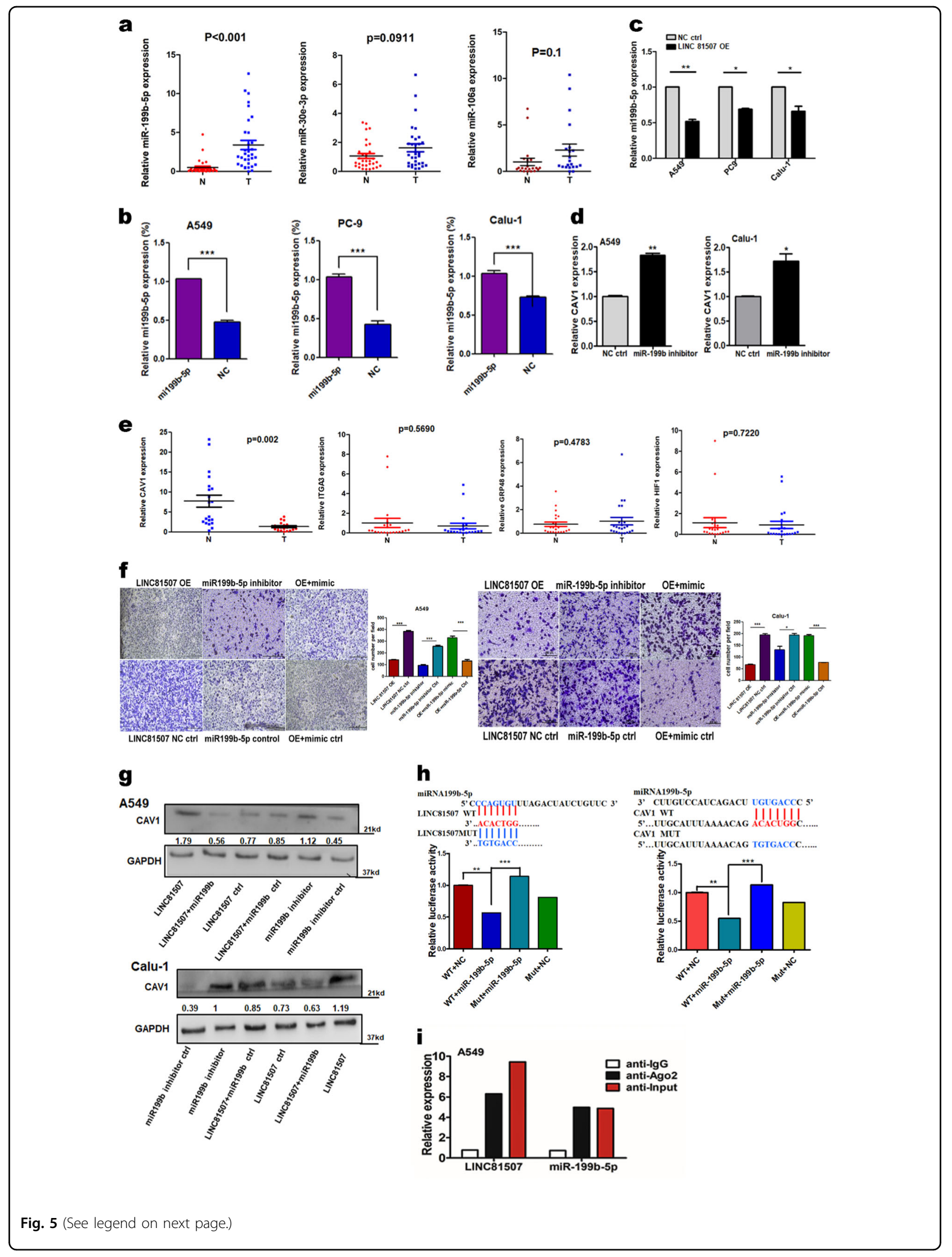


(see figure on previous page)

Fig. 5 The relation between LINC81507 and miR-199b-5p. a Relative expression of the three indicated miRNAs from more than 20 NSCLC tumor tissues and the matched adjacent normal tissues. $\mathbf{b}$ The expressions of miR-199b-5p were analyzed using RT-qPCR in NSCLC cells. c The expressions of miR-199b-5p were analyzed using RT-qPCR in NSCLC cells transfected with pCDNA3.1-LINC81507 or negative control. d The expressions of CAV1 in NSCLC cells after decreasing miR-199b-5p. e The expressions of indicated mRNAs from more than 20 NSCLC. $\mathbf{f}$ Migration assay of A549 and Calu-1 cells co-transfected with pcDNA3.1-LINC81507, negative control, miRNA-199b inhibitor or miRNA-199b-5p mimic. g Related expression of CAV1 protein in A549 and Calu-1 cells after co-transfected with pcDNA3.1-LINC81507, negative control, miRNA-199b inhibitor or miRNA-199b-5p mimic was determined by western blot analysis. $\mathbf{h}$ Schematic of LINC81507 wild-type (WT)/mutant (Mut) and pGL3-CAV1 3'-UTR Mut/WT luciferase reporter vectors are illustrated, and the relative luciferase activities were analyzed in A549 cells co-transfected with miR-199b-5p mimics or miR-NC and luciferase reporter vectors pCDNA3.1-LINC81507-WT or Mut. $\mathbf{i}$ The RIP assay revealed that LINC81507 and miR-199b-5p were enriched in the same Ago2-immunoprecipitate. Data were represented as the mean \pm SEM of three independent experiments. ${ }^{*} P<0.05,{ }^{* *} P<0.01,{ }^{* * *} P<0.001$

experiments (Fig. 6d) to find the relation between miR199b-5p and CAV1. The 3'-UTR region of CAV1 mRNA, including the predicted miR-199b-5p recognition site (CAV1-WT) or the mutated sequence (CAV1-MUT), were cloned into luciferase reporter plasmids (Fig. 5h). Luciferase activity was lower in the wild-type vector than that in the mutant vector. In addition, the RNA levels of CAV1 were decreased by miR-199b-5p (Fig. 5d). Furthermore, our data proved that CAV1 is a potential target of miR-199b-5p. Then we questioned whether the CAV1 gene is regulated by the aberrant expression of LINC81507. Overexpression of LINC81507 raised the expression of CAV1 (Fig. 4c), which was consistent with the in vivo results (Fig. 2e). Consistently, miR-199b-5p inhibitor have similar effects as LINC81507 (Fig. 5g). Similarly, inhibition of migration by CAV1 could be reversed by co-transfection of miR-199b-5p inhibitor and CAV1 ctrl (Fig. 6d). These data support our speculation that LINC81507 inhibits NSCLC metastasis through the miR-199b-5p/CAV1 axis.

\section{LINC81507/miR-199b-5p/CAV1 axis negatively regulates metastasis via STAT3 signaling}

As LINC81507/miR-199b-5p/CAV1 axis can regulate NSCLC proliferation and metastasis, we further explored the underlying signaling pathways. Research has reported that CAV1 regulates lung cancer's apoptosis via STAT3 pathway $^{27-29}$, which suggests that CAV1 mediate STAT3 pathway in NSCLC. Thus, we examined this axis in our study. First, we overexpressed and knockdown CAV1 (Fig. 6a). As expected, overexpression of CAV1 reduced phosphorylation of STAT3 in NSCLC (Fig. 6b), whereas reduced expression of CAV1 increased p-STAT3. These results suggest that CAV1 inhibits the STAT3 pathway in NSCLC.

Then, we hypothesized that LINC81507 can increase CAV1 via sequestering miR-199b-5p, and thereby inhibiting the STAT3 pathway and EMT in lung cancer cells. The results showed that when LINC81507 was overexpressed, the expression of $\mathrm{p}-\mathrm{STAT}{ }^{\mathrm{Y} 705}$ was remarkably decreased (Fig. 6b). When we transfected LINC81507 with or without Niclosamide (the inhibitor of STAT3, $0.1 \mu \mathrm{M})^{30-32}$, the expression of CAV1 in the group with Niclosamide was lower than that without Niclosamide (Fig. 6b). We also performed the rescue experiments in the LINC85107 and Niclosamide treated groups (Fig. 6c). These data implied that LINC81507 disrupts STAT3 phosphorylation via promoting CAV1 expression. Taken together, our data suggest that LINC81507/CAV1 can block the phosphorylation of STAT3 protein, and the LINC81507/miR-199b-5p/CAV1 axis regulates NSCLC cell metastasis in a STAT3-dependent manner.

\section{Discussion}

Recently, numerous LncRNAs have been found to regulate pathological processes in $\mathrm{NSCLC}^{8}$. In this study, we first discovered that LINC81507 was significantly decreased in NSCLC tissues and cell lines. LINC81507 suppressed proliferation, migration, invasion and EMT in vitro and in vivo. Our results suggest that LINC81507 acts as a tumor suppressor in NSCLC.

Compelling evidences has proven that subcellular localization determines biological function of LncRNAs ${ }^{33}$. The nuclear LncRNAs can regulate transcription factor directly $^{34}$, while the cytoplasmic LncRNAs can sponge miRNA or $\mathrm{mRNA}^{35}$. In our experiment, we found that LINC81507 mainly resides in cytoplasm, which implies it functions as ceRNA through sponging miRNAs. Our results showed that LINC81507 functions as a ceRNA through sponging miR-199b-5p, suppressing the oncogenic role of miR-199b-5p and plays a critical role in NSCLC progression.

The previous studies have shown that miR-199 has a potential in cancer therapies, because it is associated with various tumors, including prostate cancer ${ }^{36}$, breast can$\operatorname{cer}^{37}$, medulloblastoma ${ }^{38}$, hepatocellular carcinoma $(\mathrm{HCC})^{39}$ and osteosarcoma ${ }^{40}$. Our data provided the compelling evidence that miR-199b-5p expression is significantly upregulated and inversely correlated with LINC81507 expression in lung cancer cells. Overexpression of miR-199b-5p abolished the anti-metastatic ability of LINC81507. In addition, our bioinformatic 
$\mathbf{a}$

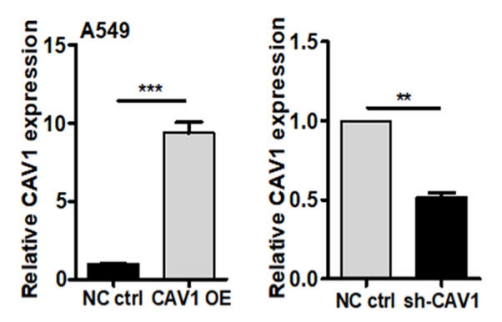

b
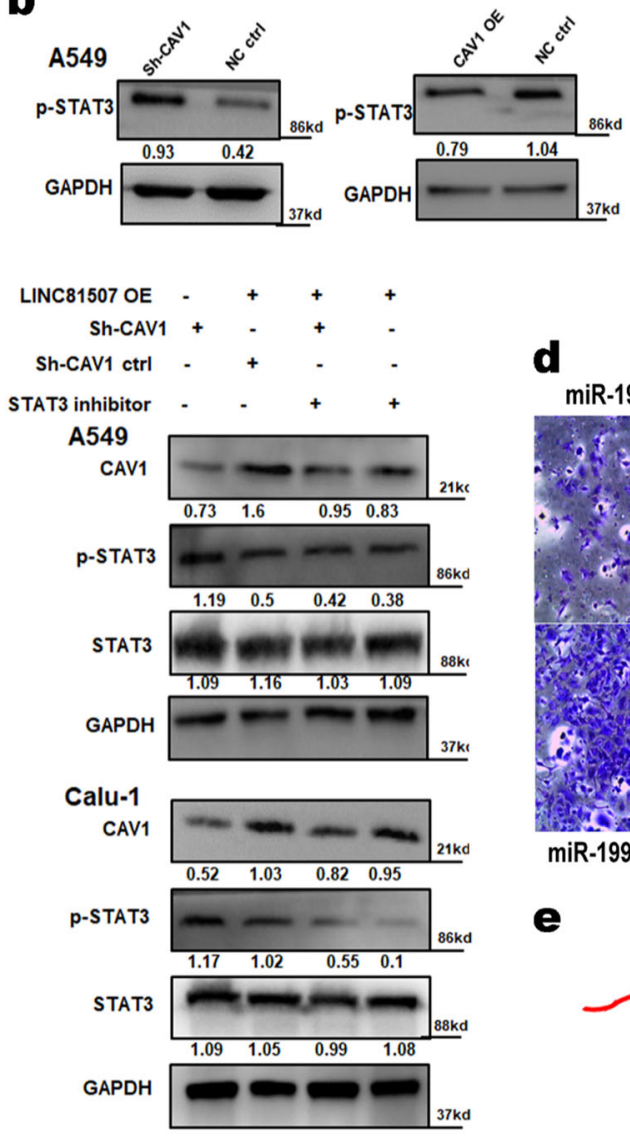

d

$\mathbf{e}$

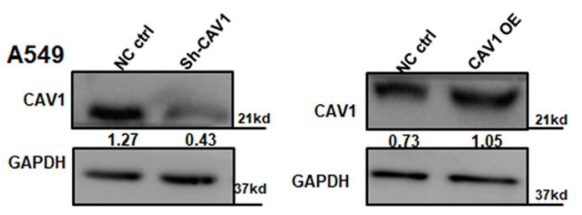

C
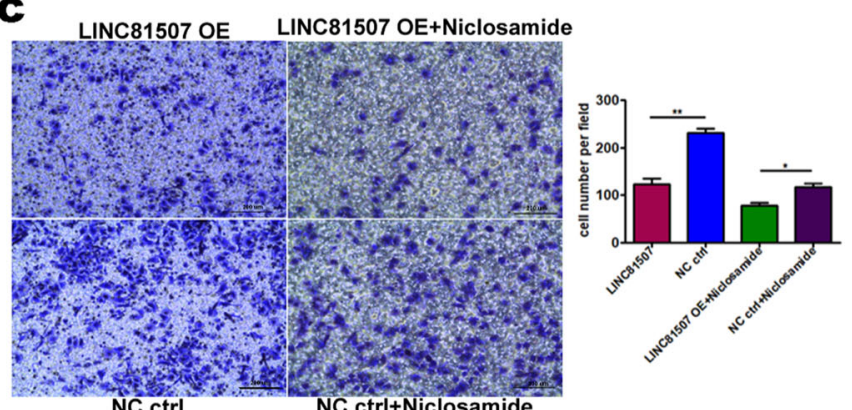

NC ctrl
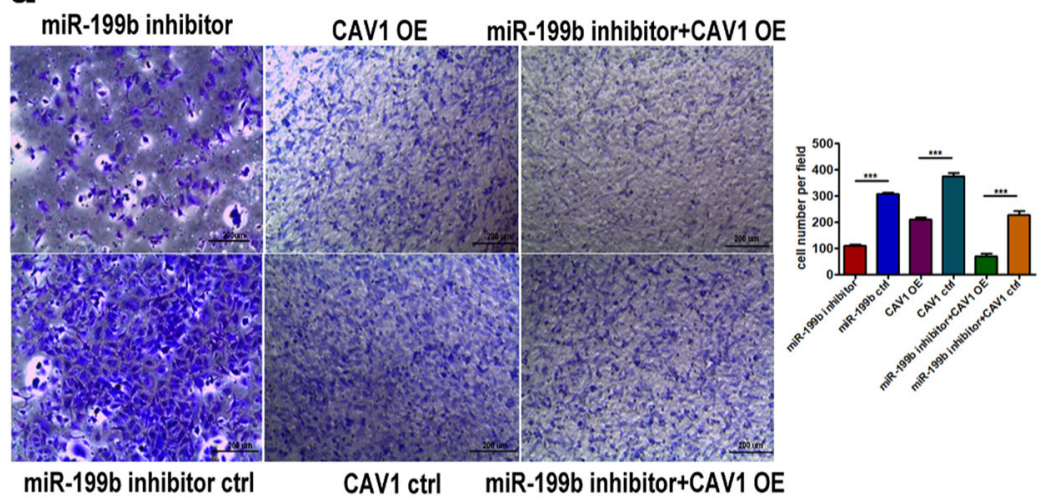

miR-199b inhibitor ctrl

CAV1 ctrl miR-199b inhibitor+CAV1 OE

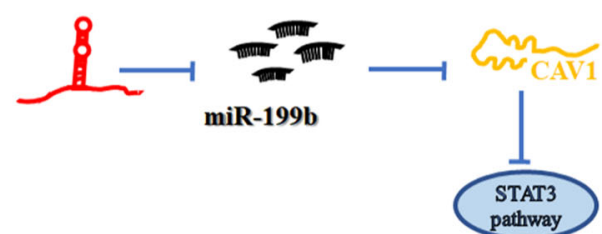

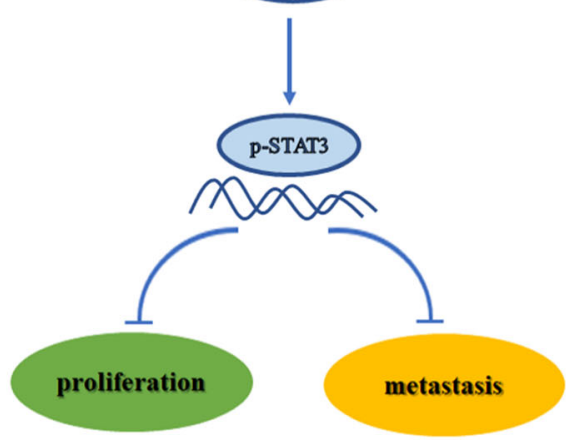

Fig. 6 In NSCLC, LINC81507/miR-199b-5p/CAV1 axis and its STAT3 pathway. a The expressions of CAV1 were determined with RT-qPCR and western blot in A549 and Calu-1 cells transfected with negative control, CAV1-overexpression, or sh-CAV1. b Western blot assay to testify the relation between LINC81507 and STAT3 pathway. c Representative images and quantification of cell migration in NSCLC cells after restrain the expression of STAT3 pathway. d Representative images and quantification of cell migration in NSCLC cells after transfection miR-199b-5p inhibitor and/or CAV1 overexpression. e The schematic diagram shows the mechanism underlying LINC81507 as a ceRNA for miR-199b-5p 
analyses, co-transfection and luciferase reporter assays demonstrated that LINC81507 can directly sponge and inhibit miR-199b-5p expression.

Recent research has revealed that through degrading or inhibiting mRNAs, miRNAs act as key signal transduction mediators in tumor signaling pathways, and regulate cell fate and function ${ }^{41-43}$. In this study, we proved that miR199b-5p targets the CAV1 gene directly. Our analysis also showed that CAV1 gene expression was inversely correlated with the LINC81507's target miRNA miR-199b-5p. Upon transfection of miR-199b-5p mimic into LINC81507-OE, the expression of CAV1 was increased in A549. Although four mRNAs (CAV1, ITGA3, GRP48, and HIF1) had a potential binding site for miR-199b-5p (data not shown) in bioinformatics analysis, only the expression of CAV1 was decreased, which suggested the specificity of miR-199b-5p binding to CAV1 gene in NSCLC.

CAV1 is a multifunctional scaffolding protein and is associated with cell surface caveolae. Current research on CAV1 indicates that CAV1 can regulate multiple cancerassociated biological processes that include tumorigenesis, tumor growth, cell migration and metastasis, cell death and survival, and multidrug resistance ${ }^{44}$. However, the function of CAV1 is still controversial. CAV1 has been reported to enhance or inhibit various processes in tumor progression, especially as a tumor suppressor in pancreatic cancer cells ${ }^{45,46}$ and NSCLC cells ${ }^{47-51}$. The controversial function of CAV1 prompted us to explore its role in our study. In our study, the expression of CAV1 was decreased in adenocarcinoma and squamous cell carcinoma, which was congruent to the expression of LINC81507. After decreasing the expression of CAV1, the inhibitory role of LINC81507 in NSCLC progression is reversed, suggesting that upregulation of CAV1 by LINC81507 contributes to the tumor suppressor role of LINC81507 in NSCLC. We first preform migration assay in miR-199b-5p and CAV1, which proved that CAV1 had the ability to decrease migration. With respect to membrane localization of CAV1, we used TEM $^{52-54}$ (transmission electron microscopy) to examine the membrane integrity after overexpression of LINC81507 in A549 cells. Compared with A549 control cell lines, A549 LINC81507-OE cell lines revealed less cell membrane damage and less alteration of cell wall structure by TEM (additional file. 7e), which need further researches.

Research showed that CAV1 can mediate lung cancer cells apoptosis through STAT3 pathway. Consistently our results suggest that the LINC81507/miR-199b-5p axis can inhibit the STAT3 signaling pathway by specifically regulate CAV1.

However, the potential mechanism underlying the lower expression of LINC81507 in NSCLC is still unclear, and what transcription factors regulate LINC81507 overexpression in NSCLC is still unknown. This warrants further investigation.

\section{Conclusions}

Collectively, this is the first report that LINC81507 is decreased in NSCLC and acts as an anti-proliferative and anti-metastatic lncRNA both in vitro and in vivo. We demonstrated that through inhibiting cell cycle progression and epithelial-mesenchymal transition (EMT), LINC81507 inhibited NSCLC cell proliferation, migration, and invasion. And it acts as a ceRNA and scaffold by sponging the oncogene miR-199b-5p, and thereby suppresses NSCLC proliferation and metastasis via CAV1/ STAT3 pathway (Fig. 6e). LINC81507 not only has a critical role in NSCLC progression but also provide a novel target for NSCLC diagnosis and treatment.

\section{Acknowledgements \\ We are grateful for all the contributions that supported this study. This work was supported by National Natural Science Foundation of China Grants (Nos. 81572281, 81702278, and 81872226). \\ Authors' contributions \\ Conceived and designed the experiments: W.P. and C.D. Performed the experiments: W.P., J.W., W.S., Y.Z., Z.P., M.D., Q.L., and Y.D. Analyzed the data: W. P., D.H., B.S., and C.D. Contributed reagents/materials/analysis tools: D.H., C.Z., Y. C., and Q.T. Wrote the paper: W.P. and C.D. All authors read and approved the final paper.}

\section{Author details \\ 'Department of Oncology, Xiangya Hospital, Central South University, Changsha 410008, PR China. ${ }^{2}$ Hunan Cancer Hospital, The Affiliated Tumor Hospital of Xiangya Medical College, Central South University, Changsha 410008 , PR China. ${ }^{3}$ College of Medicine, Washington State University Spokane, Spokane, WA 99201, USA. ${ }^{4}$ National Clinical Research Center for Geriatric Disorders, Xiangya Hospital, Central South University, Changsha 410008, PR China. ${ }^{5}$ Department of Thoracic Surgery, Xiangya Hospital, Central South University, Changsha 410008, PR China}

Data availability

All data in our study are available upon request.

Conflict of interest

The authors declare that they have no conflict of interest.

\section{Publisher's note}

Springer Nature remains neutral with regard to jurisdictional claims in published maps and institutional affiliations.

Supplementary Information accompanies this paper at (https://doi.org/ 10.1038/s41419-019-1740-9).

Received: 10 January 2019 Revised: 4 May 2019 Accepted: 13 June 2019 Published online: 11 July 2019

\footnotetext{
References

1. Bray, F. et al. Global cancer statistics 2018: GLOBOCAN estimates of incidence and mortality worldwide for 36 cancers in 185 countries. CA Cancer J. Clin. 68, 394-424 (2018)

2. Chen, W. et al. Cancer statistics in China, 2015. CA Cancer J. Clin. 66, 115-132 (2016).
} 
3. Global Burden of Disease Cancer, C. et al. The global burden of cancer 2013. JAMA Oncol. 1, 505-527 (2015).

4. Heist, R. S. \& Engelman, J. A. SnapShot: non-small cell lung cancer. Cancer Cell 21, 448 e442 (2012)

5. Vennin, C. et al. The long non-coding RNA $91 \mathrm{H}$ increases aggressive phenotype of breast cancer cells and up-regulates H19/IGF2 expression through epigenetic modifications. Cancer Lett. 385, 198-206 (2017).

6. Schmitz, S. U., Grote, P. \& Herrmann, B. G. Mechanisms of long noncoding RNA function in development and disease. Cell Mol. Life Sci. 73, 2491-2509 (2016).

7. Iyer, M. K. et al. The landscape of long noncoding RNAs in the human transcriptome. Nat. Genet. 47, 199-208 (2015).

8. Peng, Z., Zhang, C. \& Duan, C. Functions and mechanisms of long noncoding RNAs in lung cancer. Onco. Targets Ther. 9, 4411-4424 (2016).

9. Saha, P., Verma, S., Pathak, R. U. \& Mishra, R. K. Long noncoding RNAs in mammalian development and diseases. Adv. Exp. Med. Biol. 1008, 155-198 (2017).

10. Quinn, J. J. \& Chang, H. Y. Unique features of long non-coding RNA biogenesis and function. Nat. Rev. Genet. 17, 47-62 (2016).

11. Peng, W. et al. Diagnostic and prognostic potential of circulating long noncoding RNAs in non small cell lung cancer. Cell Physiol. Biochem. 49, 816-827 (2018).

12. Mao, C. et al. A G3BP1-interacting IncRNA promotes ferroptosis and apoptosis in cancer via nuclear sequestration of p53. Cancer Res. 78, 3484-3496 (2018).

13. Qi, X. et al. ceRNA in cancer: possible functions and clinical implications. J. Med. Genet. 52, 710-718 (2015).

14. Tay, Y., Rinn, J. \& Pandolfi, P. P. The multilayered complexity of ceRNA crosstalk and competition. Nature 505, 344-352 (2014).

15. Wang, M., Sun, X., Yang, Y. \& Jiao, W. Long non-coding RNA OIP5-AS1 promotes proliferation of lung cancer cells and leads to poor prognosis by targeting miR-378a-3p. Thorac. Cancer 9, 939-949 (2018).

16. Yuan, Y., Haiying, G., Zhuo, L., Ying, L. \& Xin, H. Long non-coding RNA LINC00339 facilitates the tumorigenesis of non-small cell lung cancer by sponging miR-145 through targeting FOXM1. Biomed. Pharmacother. 105 707-713 (2018).

17. Tang, Y., Xiao, G., Chen, Y. \& Deng, Y. LncRNA MALAT1 promotes migration and invasion of non-small-cell lung cancer by targeting miR-206 and activating Akt/mTOR signaling. Anticancer. Drugs 29, 725-735 (2018).

18. Peng, Z. et al. Genome-wide analyses of long noncoding RNA expression profiles in lung adenocarcinoma. Sci. Rep. 7, 15331 (2017).

19. Marbaix, E., Donnez, J., Courtoy, P. J. \& Eeckhout, Y. Progesterone regulates the activity of collagenase and related gelatinases $A$ and $B$ in human endometrial explants. Proc. Natl Acad. Sci. USA 89, 11789-11793 (1992).

20. Kalluri, R. \& Weinberg, R. A. The basics of epithelial-mesenchymal transition. J. Clin. Invest. 119, 1420-1428 (2009).

21. Thiery, J. P. \& Sleeman, J. P. Complex networks orchestrate epithelialmesenchymal transitions. Nat. Rev. Mol. Cell Biol. 7, 131-142 (2006).

22. Li, K. et al. Long non-coding RNA linc00460 promotes epithelial-mesenchymal transition and cell migration in lung cancer cells. Cancer Lett. 420, 80-90 (2018).

23. Vandooren, J., Van den Steen, P. E. \& Opdenakker, G. Biochemistry and molecular biology of gelatinase B or matrix metalloproteinase-9 (MMP-9): the next decade. Crit. Rev. Biochem. Mol. Biol. 48, 222-272 (2013).

24. Qie, S. \& Diehl, J. A. Cyclin D1, cancer progression, and opportunities in cancer treatment. J. Mol. Med. (Berl) 94, 1313-1326 (2016).

25. Li, J. et al. TANRIC: an interactive open platform to explore the function of IncRNAs in cancer. Cancer Res. 75, 3728-3737 (2015).

26. Ma, R., Wang, C., Wang, J., Wang, D. \& Xu, J. miRNA-mRNA interaction network in non-small cell. Lung Cancer. Interdiscip. Sci. 8, 209-219 (2016).

27. Chiu, W. T. et al. Caveolin-1 upregulation mediates suppression of primary breast tumor growth and brain metastases by stat3 inhibition. Cancer Res. $\mathbf{7 1}$, 4932-4943 (2011).

28. Pancotti, F., Roncuzzi, L., Maggiolini, M. \& Gasperi-Campani, A. Caveolin-1 silencing arrests the proliferation of metastatic lung cancer cells through the inhibition of STAT3 signaling. Cell Signal. 24, 1390-1397 (2012).

29. Li, L. et al. Caveolin-1-mediated STAT3 activation determines electrotaxis of human lung cancer cells. Oncotarget 8, 95741-95754 (2017).

30. Pindiprolu, S., Chintamaneni, P. K., Krishnamurthy, P. T. \& Ratna Sree Ganapathineedi, K. Formulation-optimization of solid lipid nanocarrier system of
STAT3 inhibitor to improve its activity in triple negative breast cancer cells. Drug. Dev. Ind. Pharm. 1-10, https://doi.org/10.1080/03639045.2018.1539496 (2018).

31. Li, Y. et al. Multi-targeted therapy of cancer by niclosamide: a new application for an old drug. Cancer Lett. 349, 8-14 (2014).

32. Li, R. et al. Niclosamide overcomes acquired resistance to erlotinib through suppression of STAT3 in non-small cell lung cancer. Mol. Cancer Ther. 12, 2200-2212 (2013).

33. Chen, L. L. Linking long noncoding RNA localization and function. Trends Biochem. Sci. 41, 761-772 (2016).

34. Peng, Z. et al. The long noncoding RNA LINC00312 induces lung adenocarcinoma migration and vasculogenic mimicry through directly binding YBX1. Mol. Cancer 17, 167 (2018).

35. Schmitt, A. M. \& Chang, H. Y. Long noncoding RNAs in cancer pathways. Cancer Cell 29, 452-463 (2016)

36. Leeman, M. F., Curran, S. \& Murray, G. I. New insights into the roles of matrix metalloproteinases in colorectal cancer development and progression. J. Pathol. 201, 528-534 (2003).

37. Peng, X. H. et al. MiR-124 suppresses tumor growth and metastasis by targeting Foxq1 in nasopharyngeal carcinoma. Mol. Cancer. 13, 186 (2014).

38. Gong, M. et al. miR-335 inhibits small cell lung cancer bone metastases via IGF-IR and RANKL pathways. Mol. Cancer Res. 12, 101-110 (2014).

39. Zhan, Y. et al. MiR-199a/b-5p inhibits hepatocellular carcinoma progression by post-transcriptionally suppressing ROCK1. Oncotarget 8, 67169-67180 (2017).

40. De Roock, W., De Vriendt, V., Normanno, N., Ciardiello, F. \& Tejpar, S. KRAS, BRAF, PIK3CA, and PTEN mutations: implications for targeted therapies in metastatic colorectal cancer. Lancet Oncol. 12, 594-603 (2011).

41. Bracken, C. P., Scott, H. S. \& Goodall, G. J. A network-biology perspective of microRNA function and dysfunction in cancer. Nat. Rev. Genet. 17, 719-732 (2016).

42. Zhou, Y. W. et al. miR-675-5p enhances tumorigenesis and metastasis of esophageal squamous cell carcinoma by targeting REPS2. Oncotarget 7, 30730-30747 (2016).

43. He, D. et al. Down-regulation of miR-675-5p contributes to tumor progression and development by targeting pro-tumorigenic GPR55 in non-small cell lung cancer. Mol. Cancer 14, 73 (2015).

44. Goetz, J. G., Lajoie, P., Wiseman, S. M. \& Nabi, I. R. Caveolin-1 in tumor progression: the good, the bad and the ugly. Cancer Metastasis Rev. 27, 715-735 (2008).

45. Salem, A. F. et al. Caveolin-1 promotes pancreatic cancer cell differentiation and restores membranous E-cadherin via suppression of the epithelialmesenchymal transition. Cell Cycle 10, 3692-3700 (2011).

46. Liu, L. et al. Cavin-1 is essential for the tumor-promoting effect of caveolin-1 and enhances its prognostic potency in pancreatic cancer. Oncogene 33, 2728-2736 (2014)

47. Moon, K. C. et al. Expression of caveolin-1 in pleomorphic carcinoma of the lung is correlated with a poor prognosis. Anticancer Res. 25, 4631-4637 (2005).

48. Wikman, H. et al. Caveolins as tumour markers in lung cancer detected by combined use of cDNA and tissue microarrays. J. Pathol. 203, 584-593 (2004).

49. Kato, T. et al. Difference of caveolin-1 expression pattern in human lung neoplastic tissue. Atypical adenomatous hyperplasia, adenocarcinoma and squamous cell carcinoma. Cancer Lett. 214, 121-128 (2004).

50. Fu, P. et al. The different functions and clinical significances of caveolin-1 in human adenocarcinoma and squamous cell carcinoma. Onco. Targets Ther. 10, 819-835 (2017).

51. Chen, H. L., Fan, L. F., Gao, J., Ouyang, J. P. \& Zhang, Y. X. Differential expression and function of the caveolin-1 gene in non-small cell lung carcinoma. Oncol. Rep. 25, 359-366 (2011).

52. Kopek, B. G. et al. Diverse protocols for correlative super-resolution fluorescence imaging and electron microscopy of chemically fixed samples. Nat. Protoc. 12, 916-946 (2017).

53. Attili, D. et al. Calcium-induced differentiation in normal human colonoid cultures: cell-cell / cell-matrix adhesion, barrier formation and tissue integrity. PLoS One 14, e0215122 (2019).

54. Cypriano, J. et al. Uptake and persistence of bacterial magnetite magnetosomes in a mammalian cell line: Implications for medical and biotechnological applications. PLoS One 14, e0215657 (2019). 